\title{
Coordinated Charging of Electric Vehicles for Congestion Prevention in the Distribution Grid
}

Hu, Junjie; You, Shi; Lind, Morten; Østergaard, Jacob

Published in:

IEEE Transactions on Smart Grid

Link to article, DOI:

10.1109/TSG.2013.2279007

Publication date:

2014

Link back to DTU Orbit

Citation (APA):

Hu, J., You, S., Lind, M., \& Østergaard, J. (2014). Coordinated Charging of Electric Vehicles for Congestion Prevention in the Distribution Grid. IEEE Transactions on Smart Grid, 5(2), 703-711.

https://doi.org/10.1109/TSG.2013.2279007

\section{General rights}

Copyright and moral rights for the publications made accessible in the public portal are retained by the authors and/or other copyright owners and it is a condition of accessing publications that users recognise and abide by the legal requirements associated with these rights.

- Users may download and print one copy of any publication from the public portal for the purpose of private study or research.

- You may not further distribute the material or use it for any profit-making activity or commercial gain

- You may freely distribute the URL identifying the publication in the public portal

If you believe that this document breaches copyright please contact us providing details, and we will remove access to the work immediately and investigate your claim. 


\title{
Coordinated Charging of Electric Vehicles for Congestion Prevention in the Distribution Grid
}

\author{
Junjie Hu, Student Member, IEEE, Shi You, Member, IEEE, Morten Lind, and \\ Jacob Østergaard, Senior Member, IEEE
}

\begin{abstract}
Distributed energy resources (DERs), like electric vehicles (EVs), can offer valuable services to power systems, such as enabling renewable energy to the electricity producer and providing ancillary services to the system operator. However, these new DERs may challenge the distribution grid due to insufficient capacity in peak hours. This paper aims to coordinate the valuable services and operation constraints of three actors: the EV owner, the Fleet operator (FO) and the Distribution system operator (DSO), considering the individual EV owner's driving requirement, the charging cost of $\mathrm{EV}$ and thermal limits of cables and transformers in the proposed market framework. Firstly, a theoretical market framework is described. Within this framework, FOs who represent their customer's (EV owners) interests will centrally guarantee the EV owners' driving requirements and procure the energy for their vehicles with lower cost. The congestion problem will be solved by a coordination between DSO and FOs through a distribution grid capacity market scheme. Then, a mathematical formulation of the market scheme is presented. Further, some case studies are shown to illustrate the effectiveness of the proposed solutions.
\end{abstract}

Index Terms - Congestion management, distribution grid, electric vehicles, optimal charging schedule.

\section{INTRODUCTION}

\section{A. Aggregated Charging of EVS}

$\mathbf{O}$ THER THAN fulfilling its basic transport function, EVs, as smart grid assets, can provide a large number of valuable services, e.g., meeting the balancing requirements for energy suppliers with stochastic renewables, providing regulation services to the system operators, and modifying the demand curves to defer the network expansion, etc., [1]-[7]. As a result, a new business entity, namely EV fleet operator (FO) has emerged which aims to capture the business opportunities by providing the multiple services of EVs. Alternative names for an EV FO are used such as EV virtual power plant, EV aggregator or charging service provider. The new entities could be independent or integrated in an existing business function of the energy supplier; however they all share a list of commonalities as following:

1) Same mission:

- Guarantee driving needs of the EV owners;

Manuscript received January 10, 2013; revised June 13, 2013; accepted August 13, 2013. This work is supported by Danish iPower project (http://www. ipower-net.dk/). Paper no. TSG-00019-2013.

The authors are with the Center for Electric Power and Energy, Department of Electrical Engineering, Technical University of Denmark, 2800 Kgs. Lyngby, Denmark. (e-mail: junhu@elektro.dtu.dk; sy@elektro.dtu.dk; mli@elektro.dtu.dk; joe@elektro.dtu.dk).

Color versions of one or more of the figures in this paper are available online at $\mathrm{http}: / /$ ieeexplore.ieee.org.

Digital Object Identifier 10.1109/TSG.2013.2279007
- Coordinate and support the valuable services and operation constraints of EV and power system operator;

- Maximize the renewable energy.

2) Similar methods:

- Implement centralized control/marketing method to maximize business values [1]-[7];

- Optimize the charging process of EVs [6], [8]-[11].

However, the function of the distribution grid may be challenged when FOs try to achieve these objectives, because the increasing size and number of consumption units, e.g., EVs can cause problems in peak hours [12]-[16]. Mainly two issues are considered in the literature when discussing the possible challenges in a distribution grid with increasing DERs, they are voltage drops and thermal overloading of transformers and cables. Focus in this study will be on the prevention of the thermal overloading of the transformers and cables, which is also known as congestion management. In the following of this section, we first give an overview on the centralized and market-based coordination strategies for gird congestion management. Then, the motivation and contribution of this study is presented.

\section{B. Centralized Control of EVS for Grid Congestion Management}

Lately, research done in [17]-[19] have been aiming to coordinate these multiple objectives centrally, i.e., to optimize the charging cost of EVs as well as respecting the hard constraints imposed by EV owner needs and distribution grid operation. In [17], [18], a complex scheduling problem involving EV owners, FO and DSO is analyzed. The results show that both the FO and the EV owners can achieve the objectives of minimizing charging costs and fulfilling driving requirements without violating the grid constraints. This approach requires a complex interaction between DSO and FO, but can potentially deliver a very good solution in terms of optimal grid utilization and safety. A conceptual framework consisting of both a technical grid operation strategy and a market environment is proposed in [19] to integrate EVs into the distribution systems, the activities of all the actors including the EV owner, the FO and the DSO are described and the results indicate that smart charging can maximize the EV penetration without exceeding grid constraints.

\section{Market-Based Coordination Strategies of EVs for Grid Congestion Management}

Alternatively, several ways of solving the congestion problem have been suggested from market perspective. Our previous study [20] has conceptualized several approaches to address the 
distribution grid congestion according to their value and benefits which they can offer as well as possible drawbacks and risks and the complexity of implementation. A short description of the principles behind these strategies is given below:

- Distribution grid capacity market

In this method, FOs will submit power requests to DSO for their aggregated energy/power schedule on each node (aggregated capacity); in response they will receive a price for each node which reflects the respective congestion, and are requested to update their energy schedules. The process will terminate when all constraints are satisfied. The mechanisms behind the market could be designed in many ways, such as uniform price auction mechanism [21], shadow price based mechanism [22].

- Dynamical grid tariff

In this method [23], the DSO generates a time and grid-location dependent price for grid usage based on expected nodal consumption levels. The DSO anticipates the size and the price-responsiveness of the load at critical grid nodes and calculates the price to optimally reflect the expected congestion problem. The FO will then get the dynamic nodal tariff and make an optimal schedule with respect to the predicted spot price and dynamic grid tariff.

Besides, studies in [24], [25] investigate the coordination methods using a common price signal and the work [24], [25] share a lot of similarities. Mainly, both work use game theory to formulate an EV/demand energy consumption scheduling game. The actors are assumed to be cost-minimizing and coupled via a common signal, i.e., a common electricity price in [25] and total load of the distribution grid (a heavier grid loading means a higher price) [24]. The strategies are the daily schedule of their consumption.

\section{Motivation and Contribution of This Paper}

Currently the dispatch is set only based on the day-ahead electricity market and the end-users' need for energy services. Traditionally, demand takes place when needed and the challenges in the distribution grid created by the EV aggregation could be solved by expanding the grid to fit the size and pattern of demand. As an alternative, it is assumed that the distribution grid company can benefit more by making the consumers shift demand consumption from one given period to another, after identifying the long-term marginal costs of reinforcement of the grid. Considering the new opportunity, a new scheme using both the day-ahead electricity market and the distribution grid state to set the dispatch should be established, which can enable the power system balance and the distribution grid congestion management. In general, two schemes are qualitative analyzed and proposed [12]: 1) distribution grid congestion management first, then energy system balance or 2) vice versa. Both the merits and demerits of these two strategies are well discussed in the report [12].

The present study aims to investigate the coordination strategy among DSO, FOs and EV owners based on the basic concept in [20], [12], specifically, the conceptualized proposal of "distribution grid congestion management first, then energy system balance" in [12] and the distribution grid capacity market in [20], by making them into concrete optimization

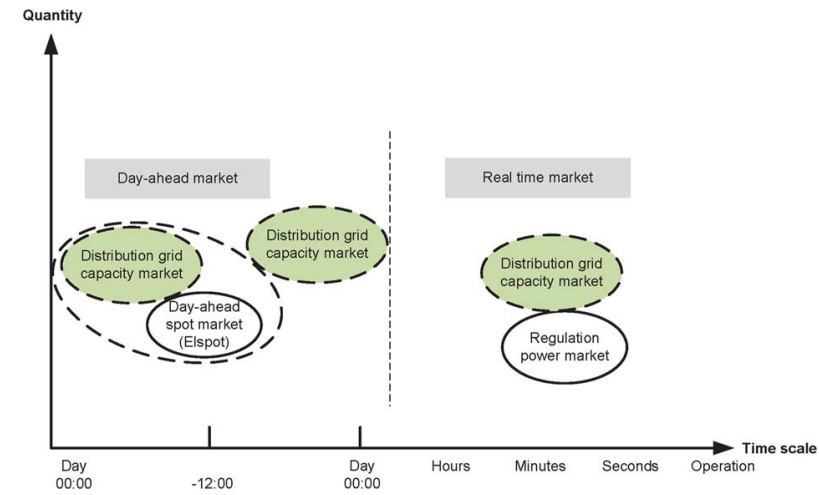

Fig. 1. The proposed distribution grid capacity market can be an integrated part of the current power markets.

problems and by showing detailed case studies. In this study, a framework is proposed which can minimize the charging cost of EVs as well as respecting to the hard constraint imposed by the EV owners and DSO. The proposed framework consists of linear programming technology based optimal charging of EVs and shadow price mechanism based distribution grid capacity market.

Fig. 1 illustrate the proposal of integrating the new proposed distribution grid capacity market into the existing power markets. It is emphasized that the market scheme is flexible and scalable, in the fig, the "distribution grid capacity market scheme" is placed in three position, which represent three different time periods, i.e., day-ahead and intra-day period for congestion prevention, real time period for congestion relief.

There are mainly two research contributions in this study. Firstly, we recommend and test a distribution grid capacity market set up enabling the distribution congestion prevention, in which multiple FOs are involved. Secondly, the proposed market framework is flexible and scalable in diverse control schemes, such as the mechanism elaborated later specifically for the day-ahead period can be also adopted for the control situation in the period of intra-day and real time, the mechanism designed for EVs can be also used for other appliances which have the capability of altering their consumption pattern with limited impact on their primary energy service, such as theoretically controlled loads.

The remainder of the paper is organized as follows: In Section II, a general explanation is given on the methodology for congestion prevention, i.e., the proposed market framework. Section III mainly presents the mathematical formulation of the congestion issues into a subgradient method based distribution grid capacity market set up. Then several case studies are illustrated in Section IV to facilitate the understanding. Finally, discussion and conclusions are made in Section V.

\section{Methodology for Congestion Prevention: System ARCHITECTURE, OPERATION PRINCIPLE}

As discussed in [26]-[28], each distribution grid has a different history, such as in some cases congestion is first expected to emerge in the medium voltage grid, while in other grids the low voltage grid is considered to be more critical. In this study, a low voltage grid is used for illustration purpose, but the proposed framework holds for medium voltage grid as well. 


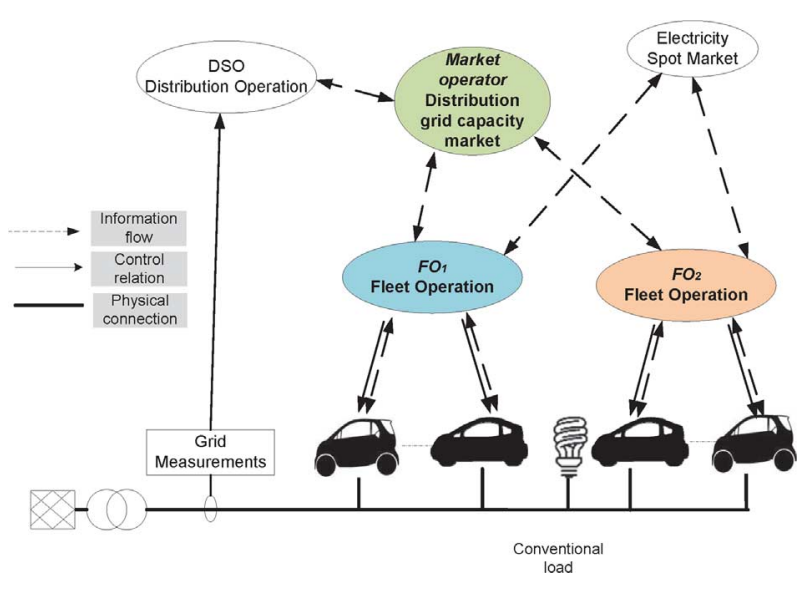

Fig. 2. A schematic of a low voltage active distribution system.

\section{A. System Architecture, Framework Design}

Fig. 2 presents a schematic of a low voltage distribution system, in which around 60-70 household consumers are connected to a 10/0.4 KV transformer (a typical Danish case), mainly connected on one feeder. In this distribution system, it is assumed that the consumers own controllable devices, i.e., EVs, besides some conventional loads, such as light or TV. These EVs are divided into two groups as illustrated in Fig. 2. One group is controlled by fleet operator-1 $\left(\mathrm{FO}_{1}\right)$, another group is controlled by fleet operator-2 $\left(\mathrm{FO}_{2}\right)$. In this hierarchical distribution system, both FOs can schedule and control their customer's electricity consumption directly. While on the FO level, the coordination between FOs and DSO is made through the distribution grid capacity market.

Within the assumed system architecture, we propose a framework consisting of four fundamental stages [20] to operate and control this system. This framework is a fully charging profile management for integrating EVs into the distribution grid smoothly.

1) Energy schedule of the FOs without congestion management-Offline scheduling. Both $\mathrm{FO}_{1}$ and $\mathrm{FO}_{2}$ need to predict the energy requirements (driving patterns) of their customers (EV owners) and plan the corresponding expected charging schedule for the EVs. The methods of estimating the energy requirements and setting up the charging schedule may be different, but in general, the FOs try to minimize the charging cost of their customers as well as guarantee the driving requirements of the $\mathrm{EV}$ owners.

2) Market based approach for distribution grid congestion prevention-Offline scheduling. The distribution grid capacity market will take effects if congestion happens. FOs trade the power capacity of the distribution grid in this market. During the negotiation of the market, a shadow price will be issued by the market operator in the time slot where congestion happens. Then this shadow price will be sent to FOs, FOs will add up the shadow price with the predicted spot price and utilize the new price to set up a new charging schedule. Again, the new schedule will be sent to the DSO/Market operator, such iteration will be terminated until the congestion is eliminated. After congestion management, FOs bid the allowable power schedule to the energy market, such as Nord Pool Spot market ${ }^{1}$ in North Europe.

3) Online scheduling and real time control. It is valuable for FOs to utilize the online scheduling stage and make better charging schemes, the general objectives are to avoid energy imbalance and to optimally participate in the regulating power market. Usually, more accurate information is provided to the FOs in this stage, FOs can judge whether they need to reschedule the charging plan based on the utility and risk analysis. With regard to real time control, one can assume that the EVs will charge according to the plan; however, if grid normal technical operation is compromised, FO management can be overridden by the DSO operation, such as using load shedding scheme.

4) Settlements. In this study, the settlements are carried out with the final energy price, i.e., the sum of spot price and shadow price (Tax, transmission, distribution fees etc., are not included here).

\section{B. Operation Principle and Assumptions}

1) $\mathrm{FO} / \mathrm{EV}$ s operation

- Aggregating EVs. From a practical perspective, it is assumed that EVs need to subscribe to one FO, maybe in the form of signing a contract that is valid for certain time period. Such subscription would possibly following the existing geographical areas, i.e., the neighborhood supplied by one FO, under one substation. The mobility of $\mathrm{EV}$, in such context, will also require the roaming-related agreement/standards among different FOs as well as an standardized ICT infrastructure, in order to make sure the FOs can access the EV information immediately when the EVs switch FOs.

- Predicting EVs driving pattern. We assume EVs have standardized function of being able to be plug and play. In most cases, they will charge at home, which is supplied by their signed FO. By establishing a database for EV users, it is feasible for FOs to predict EVs driving pattern. Besides, EV owners are encouraged to submit their draft plan for the utilization of EVs in the next day. In few cases, they may need to charge in some other areas where belong to another FO, since the fully charged battery in morning time could sustain their daily driving requirements in most times. In such case, a roaming technology widely used in the Telecom could be an example for us, which means that FO and FO can share information.

- Optimal charging schedule generation. We assume that the charging process of EVs following a linear behavior and FOs use a linear programming technology to model the optimal charging problem of EV fleet and determine the aggregated EV charging profiles. The computation speed is quite fast for FOs.

- Interacting with DSO/Market operator. For the interactions between FOs and DSO/Market operator, it is assumed that ICT infrastructure can facilitate the communication.

\footnotetext{
${ }^{1}$ http://www.nordpoolspot.com/
} 
2) DSO/Market operator operation

- Grid state estimation. The proposed solution requires a higher level of automation on the operation of distribution grid, such as demand forecasting, grid state estimation and online grid measurements. This is not an easy task for DSO at this moment, since little real time information exists about the power flow in the low voltage grid. While in the medium voltage grid, the real time measurement is traceable in the Danish distribution systems and many other European distribution systems. The good news for the low voltage grid is the installation of the smart meters, today, in Denmark, half of all households have an updated meter that can be read remotely at least on hourly basis. With these inputs, the DSO could study the co-variation between the DERs and traditional load. Data mining technology can be used to separate the information of conventional loads from EVs would be highly necessary.

- Shadow price set up. The shadow price will be determined based on the power requirement of FOs and the capacity of transformer/line of the distribution grid. More details will be presented in next section.

\section{Method Development of SMart Charging of EVs WiTH GRID CONGESTION MANAGEMENT}

In this section, algorithms and models for enabling the EV charging profile management are discussed. In short, FOs predict their customers' energy requirements and make the energy schedule, which is shown in Section III-A. Then Section III-B illustrates the method for grid congestion management. A settlement example is given in Section III-C. It is noted that the main work in this study is in the stage of offline scheduling, and it is assumed that there is no schedule changes in the online scheduling period and the EVs will charge based on the schedule made in the offline scheduling stage.

\section{A. Energy Schedule of the FOs Without Congestion Management}

We provide one solution as a reference for the energy schedule setting of FOs, the solution is based on our previous study [10], in which, an optimal charging strategy has been proposed for EVs with the purpose of fulfilling EV owner's driving requirements as well as minimizing the charging cost. The solution is briefly modified and introduced as follows:

$$
\operatorname{minimize} \sum_{i=1}^{N_{T}} \Phi_{j, i} P_{j, i} t, j=1, \ldots, N_{k}^{E}
$$

subject to

$$
\left\{\begin{array}{l}
\mathrm{SOC}_{0, j}+\sum_{i=1}^{N_{T}} P_{j, i} t_{j, i} \geq \mathrm{SOC}_{\mathrm{Min}, j}+\sum_{i=0}^{N_{T-1}} E_{\mathrm{drive}, i+1} \\
\mathrm{SOC}_{0, j}+\sum_{i=1}^{N_{T}} P_{j, i} t_{j, i} \leq w * E_{\mathrm{cap}, j}+\sum_{i=2}^{N_{T+1}} E_{\mathrm{drive}, i-1} \\
0 \leq P_{j, i} t_{j, i} \leq E_{\text {max }, j}, i=1, \ldots, N_{T}
\end{array}\right.
$$

With the above optimization problem, the FO can generate a unique energy schedule for EV owner; the sum of the individual EV energy schedule will be denoted as $P_{k, i}^{E}$, and

$$
P_{k, i}^{E}=\sum_{j=1}^{N_{k}^{E}} P_{j, i}, k=1, \ldots, N_{B}, i=1, \ldots, N_{T},
$$

where

$$
\begin{array}{ll}
N_{k}^{E} & \text { Number of EVs under FO } k . \\
N_{T} & \text { Number of time slot in the scheduling period. } \\
N_{B} & \text { Number of FOs. } \\
j & \text { Index for the number of EVs under each FO, } \\
& j=1,2, \ldots, N_{k}^{E} . \\
i & \text { Index of time slot in the scheduling period, } \\
& i=1,2, \ldots, N_{T} .
\end{array}
$$

$k \quad$ Index for the number of FOs, $k=1, \ldots, N_{B}$.

$\Phi_{j, i} \quad$ Predicted day-ahead electricity market price vector.

$P_{j, i} \quad$ Decision variable vector.

$t \quad$ Length of each time slot.

$P_{k, i}^{E} \quad$ Power requirements of EVs of each FO in each time slot.

$\mathrm{SOC}_{0, j} \quad$ Initial SOC of individual EV.

$\mathrm{SOC}_{\mathrm{Min}, j}$ Recommended minimum SOC of the EV.

Edrive The predicted individual EV owners driving requirement.

$E_{\text {max }, j} \quad$ Charge rate in term of energy of individual EV.

$w * \quad$ Recommended maximum SOC of the EV, where

$E_{c a p, j} \quad w$ is the parameter which express the charging behavior of the battery of the EV is a linear process, $E_{c a p, j}$ is the capacity of the battery of the EV.

In (1), the first constraint means that the available energy in the battery should be greater than or equal to the energy requirement for the next trip. The second constraint indicates that the available energy in the battery should be less than or equal to the power capacity of the battery. The third constraint represents that the charging rate is less than or equal to its maximum power rate of a charger. The physical meaning of the decision variable vector $P_{j, i}$ is to make a decision to distribute/charge the power on the certain time slots, where the charging cost can be minimized.

\section{B. Market Based Approach for Distribution Grid Congestion Prevention}

1) Analytical Analysis of Distribution Grid Capacity Market: In general, the method starts with a proposed cost function which represents the cost of the power preference difference of a FO in each time slot, e.g.,

$$
\mu_{k}=\zeta_{k}\left(\tilde{P}_{k, i}\right)
$$


To facilitate the understanding, we assume

$$
\mu_{k}=C_{k, i}\left(\tilde{P}_{k, i}-P_{k, i}^{E}\right)^{2},
$$

where $k, i$ keep the same with above notation, $\tilde{P}_{k, i}$ denotes the control variable, $C_{k, i}$ means the weighting factor which are associated with the power difference, the larger $C_{k, i}$ implies a smaller difference.

The objective is to minimize the cost functions as well as respect to the constraint from DSO:

$$
\operatorname{minimize} \sum_{k=1}^{N_{B}} \sum_{i=1}^{N_{T}} C_{k, i}\left(\tilde{P}_{k, i}-P_{k, i}^{E}\right)^{2}
$$

subject to

$$
\sum_{k=1}^{N_{B}} \tilde{P}_{k, i} \leq P_{\text {Cap }}(i), i=1, \ldots, N_{T},
$$

where $P_{\text {Cap }}(i)$ is the power capacity specifically for all the FOs, for example, it can be estimated by the DSO after deducting the conventional loads.

This problem is a convex optimization problem and relevant research [29], [30] show that by introducing Lagrange multipliers or shadow price $\Lambda(i) \in R^{N_{T}}$, problem (3) can be transferred into following partial Lagrangian problem:

$$
\begin{aligned}
L=\sum_{k=1}^{N_{B}} \sum_{i=1}^{N_{T}} C_{k, i}\left(\tilde{P}_{k, i}-P_{k, i}^{E}\right)^{2} & \\
+ & +\sum_{i=1}^{N_{T}} \Lambda(i)\left(\sum_{k=1}^{N_{B}} \tilde{P}_{k, i}-P_{\text {Cap }}(i)\right)
\end{aligned}
$$

The centralized optimization problem (3) is transferred into a decentralized one with associated shadow price $\Lambda(i)$ in each time slot, with the purpose of emulating the market behavior.

Following work aims to find the optimal power for each FO and the associated shadow price on the distribution grid line. We will assume, for simplicity, the Lagrangian function has a unique minimizer over $\tilde{P}_{k, i}$, which denoted as $P_{k, i}^{*}(\Lambda)$.

The dual problem (4) is then given by

$$
\begin{aligned}
\operatorname{maximize} & g(\Lambda)=\inf \left(\sum_{k=1}^{N_{B}} \sum_{i=1}^{N_{T}} C_{k, i}\left(P_{k, i}^{*}(\Lambda)-P_{k, i}^{E}\right)^{2}\right. \\
& \left.+\sum_{i=1}^{N_{T}} \Lambda(i)\left(\sum_{k=1}^{N_{B}} P_{k, i}^{*}(\Lambda)-P_{\text {Cap }}(i)\right)\right) .
\end{aligned}
$$

This dual problem (5) will be solved by projected subgradient method [31]-[33],

$$
\Lambda(i)^{\omega+1}=\Lambda(i)^{\omega}+\alpha_{\omega} \cdot S(i), i=1,2, \ldots, N_{T},
$$

where $\partial(-g)(\Lambda(i))$ is the subdifferential of $(-g)$ at $\Lambda(i)$ and $S(i) \in \partial(-g)(\Lambda(i))$, one can find $S(i)=\sum_{k=1}^{N_{B}} P_{k, i}^{*}\left(\Lambda^{*}\right)-$ $P_{\text {Cap }}(i)$ with $P_{k, i}^{*}\left(\Lambda^{*}\right)$ being the solution to the following optimization problem:

$\operatorname{minimize} \sum_{k=1}^{N_{B}} \sum_{i=1}^{N_{T}} C_{k, i}\left(\tilde{P}_{k, i}-P_{k, i}^{E}\right)^{2}+\sum_{k=1}^{N_{B}} \sum_{i=1}^{N_{T}} \Lambda(i) \cdot \tilde{P}_{k, i}$

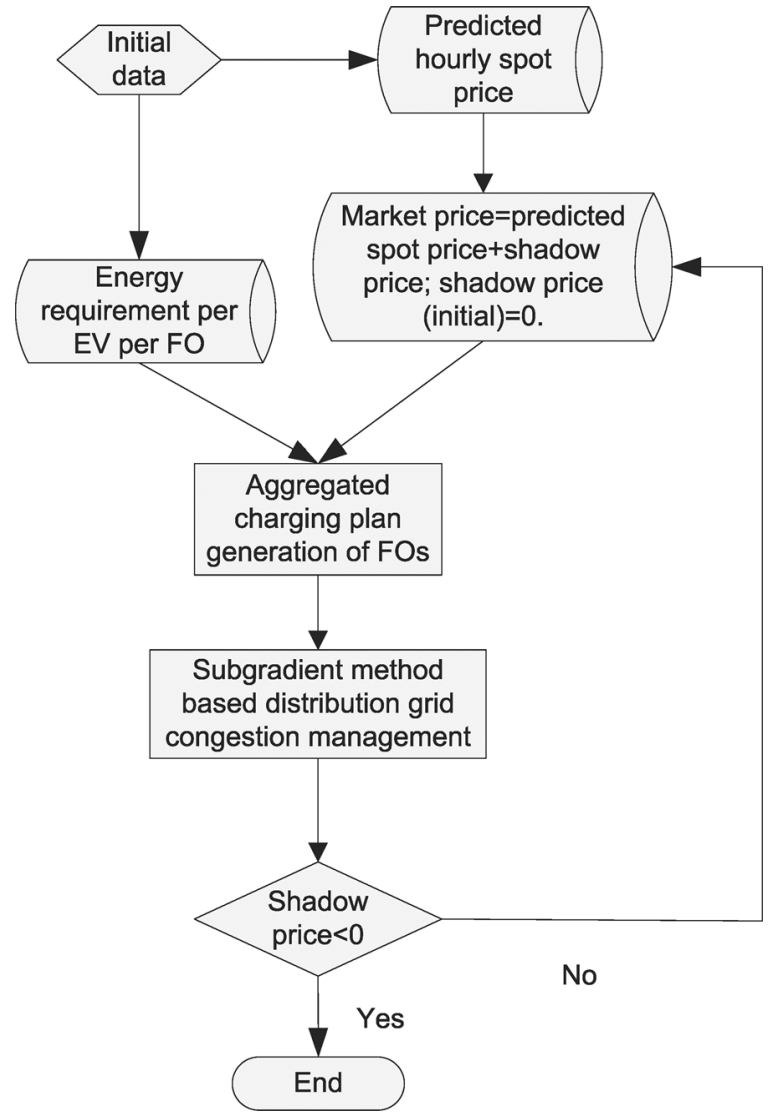

Fig. 3. Flow chart of the proposed cost and schedule adjustment algorithm.

This optimization is completely separable between various FOs, and can therefore be solved distributively. For each FO, such as $\mathrm{FO}_{k}$, the optimization problem becomes

$$
\text { minimize } \sum_{i=1}^{N_{T}} C_{k, i}\left(\tilde{P}_{k, i}-P_{k, i}^{E}\right)^{2}+\sum_{\substack{i=1 \\ k=1}}^{N_{T}} \Lambda(i) \cdot \tilde{P}_{k, i},
$$

Solving problem (8) for the FOs gives power $P_{k, i}^{*}\left(\Lambda^{*}\right)$ that can be used to find the subgradient:

$$
S(i)=\sum_{k=1}^{N_{B}} P_{k, i}^{*}\left(\Lambda^{*}\right)-P_{\mathrm{Cap}}(i)
$$

2) Cost and Schedule Adjustment Algorithm: The following steps illustrate the cost adjustment algorithm which are illustrated in Fig. 3 and can mimic the trading and negotiation process in the distribution grid capacity market, when congestion happens. The algorithms integrate the mechanisms discussed in the above of this section.

1) FOs submit their energy schedule to the distribution grid capacity market before submitting them to the electricity spot market.

2) The DSO/Market operator predicts whether congestion will happens based on the schedules of FOs, if happens, go to the distribution grid capacity market, otherwise, the energy schedule is approved.

3) Distribution grid capacity market operation 


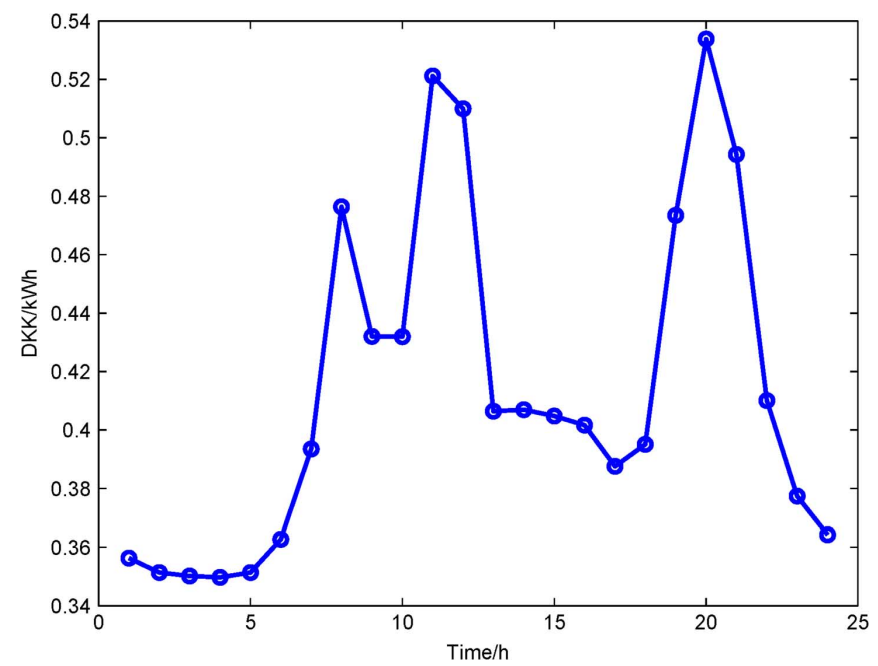

Fig. 4. Spot price in one day (12, Jan, 2011), DK-West, from NordPool, which is used as the predicted price for fleet operator.

Initialize dual variable $\Lambda(i)^{\omega}:=\Lambda_{0}(i) \geq 0$, e.g., using $\Lambda_{0}(i)=0$ or $\Lambda_{0}(i)=\Lambda(i-1)$.

loop

- With the parameter of $\Lambda(i)$, market operator determines the capacity margins $S(i)$ using (9) based on the solutions $P_{k, i}^{*}\left(\Lambda^{*}\right)$ of $(8)$.

- Update the variables $\Lambda(i)^{\omega+1}=\Lambda(i)^{\omega}+\alpha_{\omega} \cdot S(i)$, until the prices converge.

4) The new shadow price will be sent to FOs, FOs will add up this price on top of predicted spot price, recalculate the optimization problem (1), and get a new energy/power schedule $P_{k, i}^{E}$ and send to the DSO/Market Operator.

5) Do the above calculation (step 2 and 3) again, until $\Lambda(i)<$ 0 , then terminate the iteration.

6) Bid final energy/power schedule $P_{k, i}^{E}$ to the electricity spot market.

In above algorithm [31], $\alpha_{\omega} \in R$ denotes the step size and can be chosen as $\alpha_{\omega}=\alpha$ which is a positive constant, independent of $k$; with such choice, the convergence is guaranteed.

\section{Settlements}

In the settlement stage, the sum of the electricity spot price and shadow price will be used as an energy price, and the corresponding cost for FOs are given by

$$
\operatorname{Cost}(k)=\sum_{i=i}^{N_{T}}(\Phi(i)+\Lambda(i)) \cdot P_{k, i}^{\text {final }}, k=1, \ldots, N_{B} .
$$

\section{CAse Study}

In this case study, a representative distribution grid is illustrated in Fig. 2. It is assumed that 60 households are connected on the feeder. Sixty percent of the consumers are assumed to have EVs which are operated by $\mathrm{FO}_{1}$ and $\mathrm{FO}_{2}$. Specifically, $P_{\text {Cap }}$ represents the capacity of the transformer/cable and this capacity will be shared by $\mathrm{FO}_{1}$ and $\mathrm{FO}_{2}$ during the scheduling and operation period.
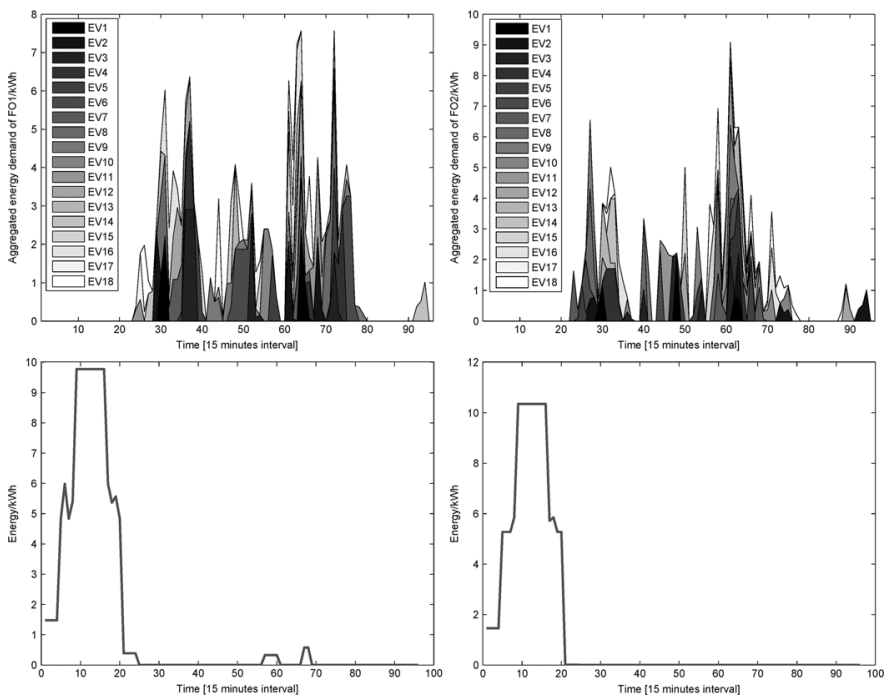

Fig. 5. Top: Aggregated energy demand of $\mathrm{FO}_{1}$ and $\mathrm{FO}_{2}$; Bottom: Aggregated energy schedule of $\mathrm{FO}_{1}$ and $\mathrm{FO}_{2}$.

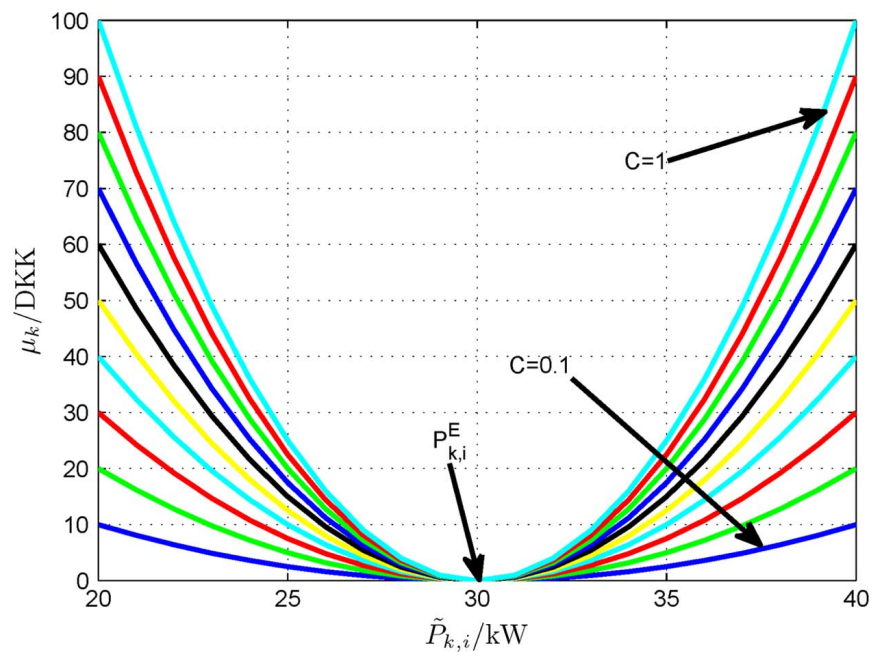

Fig. 6. A hypothetical cost function of (2), $C$ has a stepwise increase of 0.1 from 0.1 to 1 .

\section{A. Energy Schedule of the Fos Without Congestion Management}

For the EV charging schedule, the information of hourly electricity spot price of the Nordic power market is assumed to be perfectly known by the FOs, and the price data is identical with previous study [10], which is illustrated in Fig. 4. The artificial driving data of the EV fleet have been generated based on the 2003 AKTA Survey [34], in which 360 cars in Copenhagen were tracked using GPS from 14 to 100 days. Each data file includes starting and finishing time, and the corresponding duration and distance. The original data is transferred into 15 minutes interval driving energy requirements based on the assumption of $11 \mathrm{kWh} / 100 \mathrm{~km}$. The energy requirements of $\mathrm{FO}_{1}$ and $\mathrm{FO}_{2}$ are the sum of the $18 \mathrm{EVs}$, which is illustrated in the top of Fig. 5. It may not be easy to identify the individual EV's driving energy requirements, but a general trend can be concluded that most of the driving time is located in the morning and evening periods. In $\mathrm{FO}_{1}$, EV12 has the largest energy requirement which is $15.45 \mathrm{kWh}$. In $\mathrm{FO}_{2}$, it is EV4 that needs the most energy 


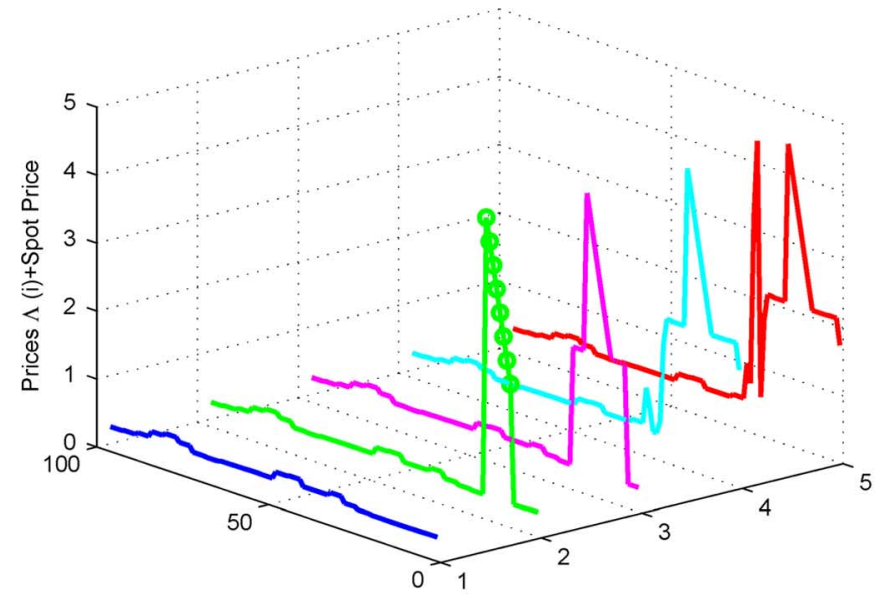

Time [15 minutes interval]

Iteration number

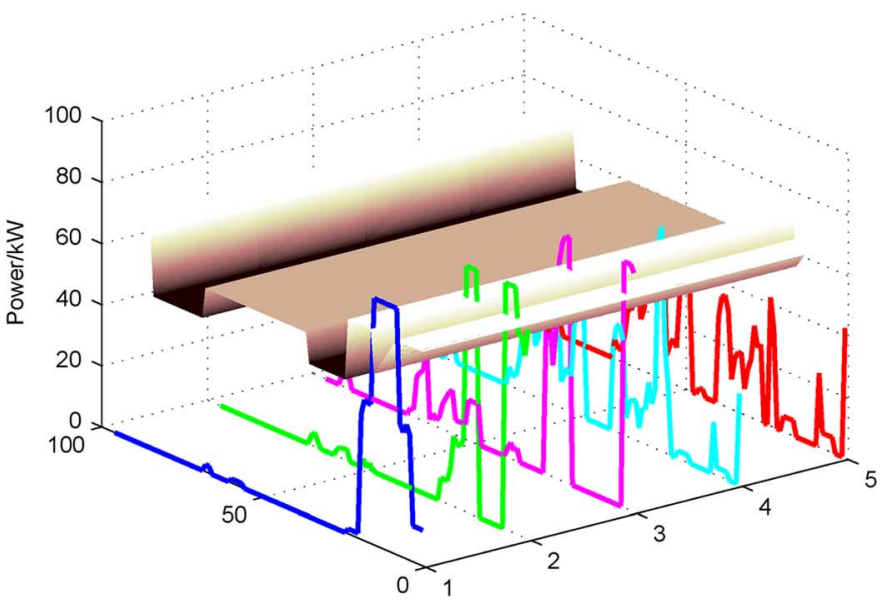

Time [15 minutes interval]

Iteration number

Fig. 7. Left: The sum of the spot price and shadow price in each iteration step. Right: The comparisons of FO's power schedule in each step with the power capacity.

which is $11.55 \mathrm{kWh}$. We assume that the data used for the simulation which represent the EV owners' driving requirements are perfectly known to the FOs. The 15 minutes interval is changeable rather than absolute. For other parameters:

- Battery capacity is set to $20 \mathrm{kWh}$

- $\mathrm{SOC}_{o}$ is set to 0.2 of the battery capacity

- $\mathrm{SOC}_{\min }$ is set to 0.2 of the battery capacity

- $\mathrm{SOC}_{\max }$ is set to 0.85 of the battery capacity

- Maximum charging power is limited to $2.3 \mathrm{~kW}$, this fits with the Danish case (10 A, $230 \mathrm{~V}$ connection).

With this information, one obtains the aggregated charging energy of each FO, which is shown at the bottom of Fig. 5. It is observed that the charging period is concentrated on the early morning time due to the lower electricity price in that time period. We assume that the power is constant in one time slot, which means the corresponding power in each time slot can be obtained $($ Energy $/ \Delta t)$.

\section{B. Market Based Approach for Distribution Grid Congestion Prevention}

In this step, we will illustrate the effectiveness of utilizing the shadow price, i.e., $\Lambda(i)$ to facilitate the congestion management in the proposed method. It is noted that the cost function in this study presented by the quadratic function is assumed to represent the cost for the energy preference loss. The accuracy of the cost function is out of the scope of this study, the focus is to show how the FOs establish the schedule based on the cost function and the shadow price. Fig. 6 illustrates the cost function of (2) with various $C_{k, i}$, in which $P_{k, i}^{E}$ is set to $30 \mathrm{~kW}$.

The power capacity $P_{\text {cap }}(i)$ is set up according to the trend in the real case; generally, the capacity is higher in the later evening and early morning time and lower in the day and evening time. The curve of the power capacity is shown by the surface in the right figure of Fig. 7. The weighting factor rate $C_{1, i}, C_{2, i}$ is set to 0.5 and 0.1 . The value of $\alpha_{\omega}$ is chosen as 0.1 in this case. Note that the variable $C_{k, i}$ and $\alpha_{\omega}$ are connected, an appropriate value of the two variables can ensure smooth operation of the proposed method, i.e., the trade-off of the speed of the convergence and the accuracy of the solution. However, there is not a strict rule for choosing the parameter values. Together with the energy schedule of $\mathrm{FO}_{1}$ and $\mathrm{FO}_{2}$ before congestion management, the values of power capacity and weighting factor rate, the simulations are presented in the Fig. 7. From these two figures, it cab be seen that the congestion problems are solved after 5 steps. Note that in the beginning, the shadow price is zero, so the blue curve represents the same price information as the one in Fig. 4. The purpose for put this price again is to get a complete view on the change of the price. Same explanation holds for the blue power curve in the right figure.

Fig. 7 presents the dynamic process of the distribution grid congestion management. It is noted that in each iteration step, the negotiation process of the FOs in the distribution grid capacity market is not shown, i.e., only the final shadow price is presented. But in order to see the effectiveness of the distribution grid capacity market, Fig. 8 is presented. In this figure, one can note the convergent process of the shadow price in the second iteration of Fig. 7. During the time slot of 9 to 16, the total power demands from $\mathrm{FO}_{1}$ and $\mathrm{FO}_{2}$ are same, but the power capacity various from $70 \mathrm{~kW}$ to $56 \mathrm{~kW}$ with a stepwise decrease of $2 \mathrm{~kW}$. The result shows that the lower power capacity results in higher shadow price. Besides, the steady state is reached quickly.

\section{Day-Ahead Congestion Settlements}

The charging cost of $\mathrm{FO}_{1}$ and $\mathrm{FO}_{2}$ are compared from two time periods, one is the cost before congestion management, and another is the cost after congestion management. Table I presents the results which show that charging cost of each FO increase a lot. It indicates a shortage of the distribution capacity.

\section{DisCUSSION AND CONCLUSION}

In this paper, two control issues are integrated in a low voltage active distribution system consisting of three actors, $\mathrm{DSO} /$ Market operator, FO and EV owner. One is the optimal charging of EVs, another is the congestion management on the distribution system level. Two steps are adopted to address these two issues, linear programming is firstly used to model the charging process of EVs and to produce an aggregated energy schedule of FOs. If the sums of the energy schedule of 


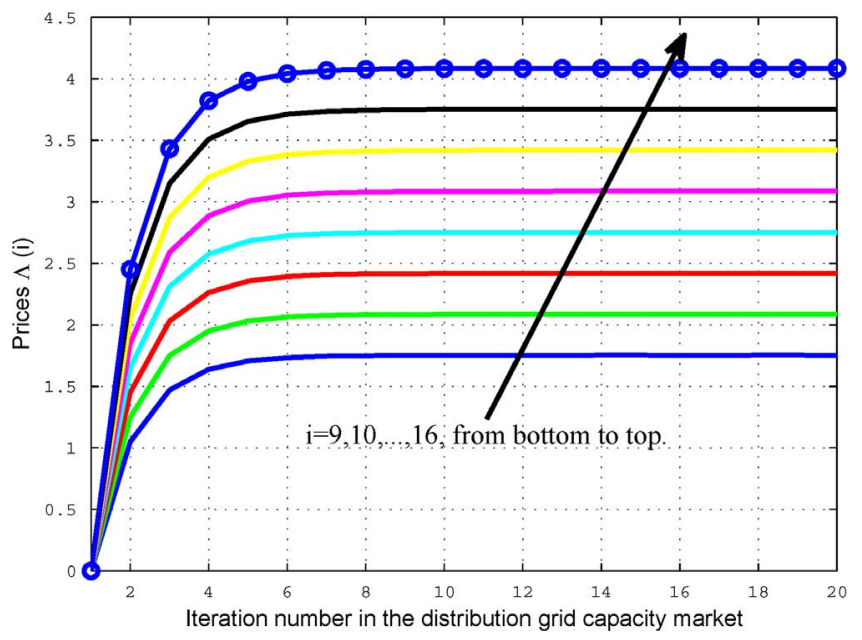

Fig. 8. Convergence of $\Lambda(i), i=9,10, \ldots, 16$ toward the shadow price in the second iteration of Fig. 7, the converged price in each time slot of this figure corresponds to the circled values in the Fig. 7(-to left).

TABLE I

Charging Cost of FOs in the Period of Before and After Congestion MANAGEMENT

\begin{tabular}{|l|l|l|}
\hline Fleet operator & $\begin{array}{l}\text { Cost before congestion } \\
\text { management }\end{array}$ & $\begin{array}{l}\text { Cost of congestion pre- } \\
\text { vention }\end{array}$ \\
\hline $\mathrm{FO}_{1}$ & $46.05 \mathrm{DKK}$ & $81.45 \mathrm{DKK}$ \\
\hline $\mathrm{FO}_{2}$ & $46.46 \mathrm{DKK}$ & $86.72 \mathrm{DKK}$ \\
\hline
\end{tabular}

the FOs overload the distribution grid, then, a distribution grid capacity market scheme is adopted to coordinate the energy schedule. The proposed solution for solving the congestion problem and managing the charging of EVs is an integration of a direct control and a price-based coordination. It is believed that the safety operation of the distribution grid can be highly ensured by coordinating the relation between the three market actors with the proposed framework. We also expect that such coordination strategy can be used to control other smart appliances, including thermostatically controlled loads such as heat pumps. As we discussed before, the market scheme can also be used in the real time for congestion relief.

We want to point out that voltage control is also an important issue for distribution grid operation, although we did not consider it in this study. In a practical way, in the planning period, DSO considers and pre-handles it by reinforcing the grid infrastructure based on the regulations already existed which describe the allowed voltage safety bands in the distribution grid. In the normal operation period, in the substation level, transformer has a tap change which can be used to regulate the voltage. Such as in Denmark, in general, $60 \mathrm{KV} / 10 \mathrm{KV}$ transformer is an OLTC (On-load tap-changers). In the future, system operator could set up some grid codes for DERs, requiring the DERs to have their own embedded voltage control, which could solve the problem preventively. In the context of this study, voltage control can also be implemented by market scheme. This approach would be to establish a market where voltage stabilizing services can be bought or sold. Technically, it is feasible. However, it is not easy to identify and validate the committed power from the DERs, which will bring many challenges. Besides, the AC power flow calculation will also introduce time-consuming problem to this method. In a simple market way, due to the increasing penetration of distributed generation, DSO can solve this problem by a contract-based solution, such as DSO can sign some contracts with FOs to get the required services.

It is noted that market approach has been well discussed and considered as one of the best approach for resource allocation, meanwhile, we also see some practical point deserve discussion for utilizing this approach for congestion management, mainly from three perspectives: 1) Stakeholder's acceptance on using price coordination: Although using price coordination approach would possibly enable an optimal resource allocation, but the uncertain in terms of end-user involvement, clear business models for FOs and DSOs, and the necessity for the regulatory support makes using price as a coordination tool for serving grid services a challenging task. 2) Size of the market and the associated market power issues: To ensure enough competition and fairness of the capacity market, one prerequisite is the number of market participants, i.e., FOs. If there are few FOs in the distribution area, issue e.g., market power will become a major challenge from the market perspective. 3) The information communicating between various stakeholders and the supporting ICT infrastructure: FOs need to communicate well with EVs in order to make an optimal charging schedule, these information include driving pattern, state of charge, some other preferences of EV owners. The time-stringent is not an essential issue here, however, EV owners cooperation are much wanted. For the interaction between FOs and distribution grid capacity market operator, real time communication is a challenge, but we can set up a reasonable time range and also try to limiting the market iteration with certain rules. This kind of setup will require advanced ICT infrastructure.

To sum up, the proposed method is flexible and scalable and can technically be enhanced to provide a complete set up for the congestion prevention in the scheduling period and congestion relief in real time, by taking into account the discussion above. Additional, the practical points discussed above imply the economic feasibility should also be analyzed in future.

\section{ACKNOWLEDGMENT}

The authors would like to thank our partners of WP4, iPower project for the discussion and inspiration. Besides, the authors are grateful to their colleagues K. Heussen and P. B. Andersen for the discussion on the grid congestion management. Also, the authors are grateful to the financial support of the Danish iPower project.

\section{REFERENCES}

[1] F. Tuffner and M. Kintner-Meyer, "Using electric vehicles to meet balancing requirements associated with wind power," Pacific Northwest National Laboratory (PNNL), Richland, WA, USA, Tech. Rep., 2011.

[2] J. Pecas Lopes, P. Rocha Almeida, and F. Soares, "Using vehicle-togrid to maximize the integration of intermittent renewable energy resources in islanded electric grids," in Proc. 2009 IEEE Int. Conf. Clean Electr. Power, pp. 290-295.

[3] J. Lopes, F. Soares, P. Almeida, and M. da Silva, "Smart charging strategies for electric vehicles: Enhancing grid performance and maximizing the use of variable renewable energy resources," in Proc. 24th Int. Battery, Hybrid, Fuel Cell Elect. Veh. Symp. Exhib. (EVS24), Stavanger, Norway, 2009.

[4] W. Kempton and J. Tomic, "Vehicle-to-grid power fundamentals: Calculating capacity and net revenue," J. Power Sources, vol. 144, no. 1, pp. 268-279, 2005. 
[5] W. Kempton and J. Tomic, "Vehicle-to-grid power implementation: From stabilizing the grid to supporting large-scale renewable energy," J. Power Sources, vol. 144, no. 1, pp. 280-294, 2005.

[6] N. Rotering and M. Ilic, "Optimal charge control of plug-in hybrid electric vehicles in deregulated electricity markets," IEEE Trans. Power Syst., vol. 26, no. 3, pp. 1021-1029, Aug. 2010.

[7] S. Han, S. Han, and K. Sezaki, "Development of an optimal vehicle-to grid aggregator for frequency regulation," IEEE Trans. Smart Grid, vol. 1, no. 1, pp. 65-72, 2010.

[8] O. Sundström and C. Binding, "Optimization methods to plan the charging of electric vehicle fleets," in Proc. Int. Conf. Control, Commun., Power Eng., pp. 28-29.

[9] T. Kristoffersen, K. Capion, and P. Meibom, "Optimal charging of electric drive vehicles in a market environment," Appl. Energy, 2010.

[10] J. Hu, S. You, J. Oestergaard, M. Lind, and Q. Wu, "Optimal charging schedule of an electric vehicle fleet," in Proc. 2011 46th Int. Universities' Power Eng. Conf. (UPEC), pp. 1-6.

[11] T. Lan, J. Hu, G. Wu, S. You, L. Wang, and Q. Wu, "Optimal charge control of electric vehicles in electricity markets," in Part of: Proc. 4th Int. Workshop Intell. Inf. Manage. Syst. Technol., 2011.

[12] C. Sndergren, "Edison project: Electric vehicles in future market models," Danish Energy Association, Tech. Rep., 2011.

[13] G. Heydt, "The impact of electric vehicle deployment on load management strategies," IEEE Trans. Power App. Syst., vol. 102, no. 5, pp. 1253-1259, 2007.

[14] R. Green, II, L. Wang, and M. Alam, "The impact of plug-in hybrid electric vehicles on distribution networks: A review and outlook," Renewable Sustain. Energy Rev., vol. 15, no. 1, pp. 544-553, 2011.

[15] J. Lopes, F. Soares, and P. Almeida, "Identifying management procedures to deal with connection of electric vehicles in the grid," Proc. 2009 IEEE Bucharest PowerTech, pp. 1-8.

[16] K. Clement-Nyns, E. Haesen, and J. Driesen, "The impact of charging plug-in hybrid electric vehicles on a residential distribution grid," IEEE Trans. Power Syst., vol. 25, no. 1, pp. 371-380, 2010.

[17] O. Sundstrom and C. Binding, "Planning electric-drive vehicle charging under constrained grid conditions," in Proc. 2010 IEEE Int. Conf. Power Syst. Technol. (POWERCON), pp. 1-6.

[18] O. Sundstrom and C. Binding, "Flexible charging optimization for electric vehicles considering distribution grid constraints," IEEE Trans. Smart Grid, vol. 3, no. 1, pp. 26-37, 2012.

[19] J. Lopes, F. Soares, and P. Almeida, "Integration of electric vehicles in the electric power system," Proc. IEEE, vol. 99, no. 1, pp. 168-183, 2011.

[20] P. B. Andersen, J. Hu, and K. Heussen, "Coordination strategies for distribution grid congestion management in a multi-actor, multi-objective setting," in Proc. 2012 Eur. ISGT, Berlin, Germany.

[21] R. D. Zimmerman, "Uniform price auctions and optimal power flow," Matpower Technical Note, Feb. 1, 2010 [Online]. Available: http:// www.pserc.cornell.edu/matpower/TN1-OPF-Auctions.pdfF

[22] B. Biegel, P. Andersen, J. Stoustrup, and J. Bendtsen, "Congestion management in a smart grid via shadow prices," in Proc. 8th IFAC Symp. Power Plant Power Syst. Control, Toulouse, France, Sep. 2012.

[23] N. O'Connel, Q. Wu, J. Stergaard, A. Nielsen, S. Cha, and Y. Ding, "Electric vehicle (ev) charging management with dynamic distribution system tariff," in Proc. 2011 ISGT.

[24] A. Mohsenian-Rad, V. Wong, J. Jatskevich, R. Schober, and A. LeonGarcia, "Autonomous demand-side management based on gametheoretic energy consumption scheduling for the future smart grid," IEEE Trans. Smart Grid, vol. 1, no. 3, pp. 320-331, 2010.

[25] Z. Ma, D. Callaway, and I. Hiskens, "Decentralized charging control for large populations of plug-in electric vehicles," IEEE Trans. Control Syst. Technol., vol. 21, no. 1, pp. 67-78, 2013.

[26] A. Ipakchi and F. Albuyeh, "Grid of the future," IEEE Power Energy Mag., vol. 7, no. 2, pp. 52-62, 2009.

[27] C. B. Lotte, H. Rasmussen, and M. Togeby, "Managing congestion in distribution grids-market design consideration," Ea Energy Analyses, Tech. Rep., 2012.

[28] M. D. Galus, C. Dobler, R. A. Waraich, G. Andersson, R. A. Waraich, R. A. Waraich, G. Andersson, and G. Andersson, "Predictive, distributed, hierarchical charging control of PHEVs in the distribution system of a large urban area incorporating a multi agent transportation simulation," ETH, Eidgenössische Technische Hochschule Zürich, IVT, Institut für Verkehrsplanung und Transportsysteme, 2011.
[29] S. Boyd and L. Vandenberghe, Convex Optimization. Cambridge, U.K.: Cambridge Univ. Press, 2004.

[30] S. Boyd, L. Xiao, A. Mutapcic, and J. Mattingley, "Notes on decomposition methods," Notes for EE364B, Stanford Univ., 2007.

[31] S. Boyd, L. Xiao, and A. Mutapcic, "Subgradient methods," Lecture notes of EE392o, Stanford Univ., Autumn Quarter, vol. 2004, 2003.

[32] M. Grant, S. Boyd, and Y. Ye, "CVX users guide," Tech. Rep., Technical Report Build 711, 2009 [Online]. Available: http://citeseerx.ist. psu.edu/viewdoc/download

[33] M. Grant and S. Boyd, "Cvx: Matlab software for disciplined convex programming," 2008 [Online]. Available: http://cvxr.com/cvx/

[34] L. Christensen, "Edison Project Report: Wp1.3 Electric vehicles and the customers," Tech. Rep., DTU Transport, 2011.

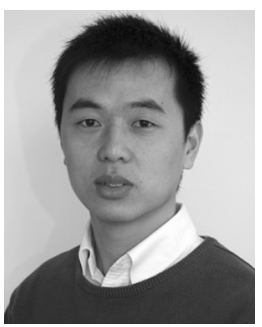

Junjie Hu received the Master of Engineering in control theory and control engineering from TongJi University, Shanghai, China, in 2010. Currently, he is a Ph.D. student at the Department of Electrical Engineering within the Technical University of Denmark (DTU). His Ph.D. project focuses on integrating control policies on controllable load, mainly electric vehicle, for active power distribution system, of which the control policies are direct control and indirect (price) control.

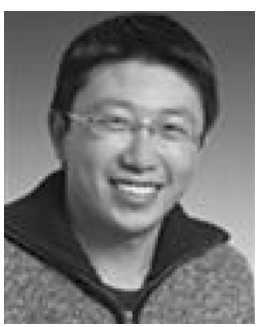

Shi You received the M.Sc. degree in electrical engineering from Chalmers Institute of Technology, Sweden, and the Ph.D. degree in electrical engineering from Technical University of Denmark, in 2006 and 2011, respectively. He is currently a Postdoctoral Researcher with the Department of Electrical Engineering, Technical University of Denmark. His main fields of interest are renewable energy integration, distribution grid planning, and electricity markets.

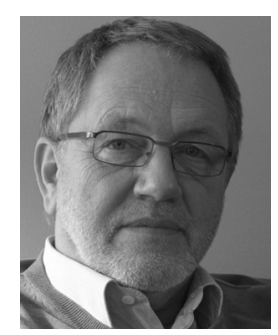

Morten Lind is Professor Emeritus of Automation at Department of Electrical Engineering at Technical University of Denmark and is associated with the Centre for Electric Power and Energy. His research interests include automation design, supervisory control of complex industrial systems and infrastructures, functional modeling, and application of agent technology and knowledge based system in automation.

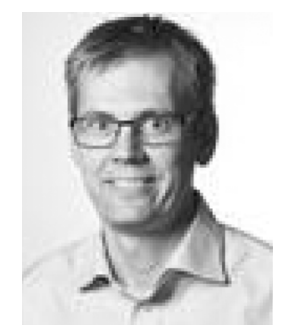

Jacob Østergaard (M'95-SM'09) received the M.Sc. degree in electrical engineering from the Technical University of Denmark (DTU), Lyngby, Denmark (DTU), in 1995.

$\mathrm{He}$ is a Professor and Head of the Centre for Electric Power and Energy, Department of Electrical Engineering at DTU. From 1995 to 2005, he worked for the Research Institute for the Danish Electric Utilities. His research interests include system integration of wind power, control architecture for future power systems, and demand side.

Prof. Østergaard is serving in several professional organizations including the EU SmartGrids advisory council. 